\title{
Inductively-Coupled Plasma Optical Emission Spectrometer
}

National Cancer Institute

\section{Source}

National Cancer Institute. Inductively-Coupled Plasma Optical Emission Spectrometer.

NCI Thesaurus. Code C62338.

A spectrometer in which a sample is introduced, either by spraying or ultrasonic nebulization, into inductively-coupled plasma generated from argon gas. This process excites dissolved metals, thereby causing them to emit radiation in both the UV and visible spectrum. The emitted radiation is dispersed by a grating monochrometer and detected with photomultiplier tubes. 